\title{
Implementation of Pre-Operative Checklist: An Effort to Reduce Delays in Surgery and to Improve Patient Safety
}

\author{
Diane Muhimpundu ${ }^{1}$, Mediatrice Batangana ${ }^{1}$, Egide Iyamuremye ${ }^{1}$, Dancilla Nyirasebura ${ }^{1}$, \\ Rosie Borey ${ }^{1}$ \\ ${ }^{1}$ University Teaching Hospital of Kigali, Rwanda
}

\section{Background}

A quality improvement project conducted at a teaching hospital in Rwanda in October 2013, showed the operating theatres were underutilized, with delayed surgery start times and prolonged turnover. One factor contributing to these delays was the inadequate preparation of patients prior to transfer to the theatre department.

\section{Description}

Multidisciplinary stakeholders from the departments of anesthesia, surgery and nursing provided valuable insight to develop a pre-operative checklist to ensure that patients were prepared for surgery and to minimize disruptions to patient flow. Both the World Health Organization surgical safety guidelines and safety checks within the Joint Commission's universal protocol, such as surgical site marking, were reviewed and incorporated into the checklist. The checklist was implemented in December 2013 and a corresponding policy was also developed and implemented. Education regarding the completion of the checklist and its importance was conducted both in the theatre and in departments that frequently transfer patients to the theatre. A retrospective documentation audit was conducted in May 2014, showing 59\% compliance in completing the checklist. Since the audit, further education has been provided to staff in attempts to increase the compliance rate.

\section{Lesson Learned}

Successful implementation was dependent on the involvement of a multidisciplinary team, and ongoing education regarding each component of the checklist. The checklist has helped to standardize perioperative care delivery, and improve overall interdisciplinary communication.

\section{Conclusion}

Patient safety should be a top priority of every operating theatre. Implementation of a standardized preoperative checklist at CHUK has shown to be an innovative tool for improving patient preparation, communication and overall efficiency in the operating room.

Key words: preoperative checklist, operating theatre, patient safety, multidisciplinary collaboration 